\title{
Probes of shape transitions from mass and charge radii of nuclear ground states
}

\author{
B.H. Sun ${ }^{1, a}$ and C.Y. Liu ${ }^{1}$ \\ ${ }^{1}$ School of Physics and Nuclear Energy Engineering, Beihang University, Beijing 100191, China
}

\begin{abstract}
The masses and sizes of nuclear ground states constitute two of the most precise and extensive arrays of experimental information. These data make a model-independent view of microscopic nuclear structure possible. Relevant differential observables of nuclear mass and charge radius can be highly sensitive to nuclear shape transitions. In this contribution, we examine the correlation of these two bulk properties to nuclear shape transitions. By combining different observables, it is even possible to isolate shape transitions from nuclear shell closures.
\end{abstract}

\section{Introduction}

One of the goals of nuclear physics is to understand how structure evolves in atomic nuclei with changing numbers of their proton and neutron constituents. This investigation dates back to the early days of the field. It was fundamental, for example, to the development of the shell model with the identification of magic numbers, and also to the earliest studies of collective structure. Today it remains one of the backbones for nuclear structure studies, especially in rare nuclei, far from the valley of stability. Because of its major role in the classification of nuclei, the unexpected onset of nuclear deformation and shape coexistence are very important. Experimentally, searching for unambiguous evidence of shape transitions or coexistence is thus crucial to reveal the single-particle level and structure changes $[1,2]$.

Both the mass and size of an atomic nucleus are bulk properties that reflect the total effect of all nucleons inside and their interactions [3-5]. Rearrangements of nucleons in nuclei due to, for example, the onset of deformation, or changes in single-particle levels, may manifest as subtle changes in these ground-state properties. Moreover, it is possible to magnify the subtle changes by using selected differential properties of nuclear masses and sizes. Just as in nuclear spectroscopic studies that use excited level structure, the measurement of nuclear masses and sizes to ultra-high precision can be a sensitive probe of sudden changes in the nuclear surface. Correlations of different observables in both ground states and excited states have been found (see e.g. [6]).

Considering that both the quality and quantity of experimental data on nuclear ground-state properties have increased tremendously over the last decade, it becomes increasingly useful to evaluate the systematic correlation

\footnotetext{
a e-mail: bhsun@buaa.edu.cn
}

between mass, size and shape transitions. In many cases, these two properties have been studied only recently for rare isotopes. In this work, we limit our investigation to nuclear ground-state properties, in particular the nuclear charge radius, which reflects in particular the distribution of protons inside an atomic nuclide. For this purpose, we take several isotopic chains at $Z \sim 40,62$ and 82 as examples to illustrate the different probes of shape transitions. In this work, we refer to shape transitions quite generally as effects induced by changes in nuclear shapes, e.g., shape coexistence. For the sake of completeness, we will also show spectroscopic observables for comparison.

This paper is organized as follows. In Section 2, we first show the finite-difference formula that will be used to extract the effect of nuclear shape transition. Then in Section 3, we examine the signatures of shape coexistence from both mass and charge radius for three different zones in nuclear chart. Finally we give a summary in Section 4.

\section{Probes for nuclear shape variances}

We first discuss the key observables that will be used to identify shape transitions. Unless otherwise indicated, the data are taken from the the most recent completions of atomic mass and charge radius evaluations [7, 8]. Energies of the first-excited $2^{+}$states $\left[E\left(2_{1}^{+}\right)\right]$and the transition probabilities between the first $2^{+}$and $0^{+}$ground states $[B(E 2)]$ are from Ref. [9].

In principle, except where there is shell closure, phase transition, or neutron-proton pairing, or where the Wigner effect is present, mass or charge radii are a smooth function of $Z$ and $N$. Therefore, finite-differences of masses and charge radii are a convenient means to identify subtle changes induced by variances in nuclear structure.

Two-neutron separation energies $S_{2 n}(Z, N)$ are frequently used to avoid oscillations between odd and even 
nuclei due to pairing. Other finite-difference formula of nuclear masses used in this work include the effective neutron shell gap $G_{2 n}(Z, N)$ and the empirical interaction strength between last two valence neutrons and two protons $\delta V_{2 p-2 n}(Z, N)$. These two quantities can be understood in the framework of the independent particle shellmodel. They are defined as:

$$
\begin{aligned}
& G_{2 n}(Z, N)= S_{2 n}(Z, N)-S_{2 n}(Z, N+2) \approx 4 \frac{\partial M^{2}(Z, N)}{\partial N^{2}} \\
& \delta V_{2 p-2 n}(Z, N)=-\frac{1}{4}[M(Z, N)+M(Z-2, N-2)\quad-M(Z, N-2)-M(Z-2, N)] \\
& \approx-\frac{1}{4} \frac{\partial M^{2}(Z, N)}{\partial N \partial Z}
\end{aligned}
$$

where $M(Z, N)$ is the mass of nuclide $(Z, N) . \delta V_{2 p-2 n}$ can be generalized to $\delta V_{i p-j n}$ between the last $i$ proton(s) and the last $j$ neutron(s) [10-14].

As for nuclear charge radii $R_{c h}(Z, N)$, similar difference formula exist. The kink strength $S(Z, N)$ is the second differential of the mean-square charge radius:

$S(N)=[R(N+2)-2 R(N)+R(N-2)] A^{2 / 3} \approx 4 \frac{\partial^{2} R(N)}{\partial N^{2}} A^{2 / 3}$,

where the $A^{2 / 3}$ term accounts for the mass dependence. Very recently, we proposed a new set of differential relations $\delta R_{i p-j n}$ based on neighboring nuclei [15]:

$$
\begin{aligned}
\delta R_{i p-j n}(Z, N)= & R(Z, N)+R(Z-i, N-j) \\
& -R(Z, N-j)-R(Z-i, N) \\
\propto & \frac{\partial R^{2}(Z, N)}{\partial N \partial Z} .
\end{aligned}
$$

Calculation of $\delta R_{i p-j n}(Z, N)$ for a single nuclide $(Z, N)$ will be done in three different forms, i.e., $\delta R_{1 p-1 n}(Z, N)$, $\delta R_{1 p-2 n}(Z, N)$, and $\delta R_{2 p-1 n}(Z, N)$. When several predictions exist for a specific nuclide, the mean value $\overline{\delta R}_{p-n}(Z, N)$ is then calculated and treated afterwards for this nuclide.

\section{Selected examples at ( $Z \sim 40, N \sim 60)$, $(Z \sim 64, N \sim 90)$ and $(Z \sim 82)$}

Here we show the ground state and excited states observables as function of neutron number, namely, two-neutron separation energies, charge radii, $\delta V_{2 p-2 n}, \overline{\delta R}_{p-n}, E\left(2_{1}^{+}\right)$, and $B(E 2)$, for three selected zones characterized by nuclear shape changes. Because there are similarities in the following discussions of selected zones, it is useful first to summarize the general features of different observables at occurrences of changes in nuclear structure.

$S_{2 n}$ decreases smoothly with neutron number between two neutron magic numbers, and deviations from this behavior is a manifestation of changes in microscopic nuclear structure. For example, a sharp decrease of $S_{2 n}$ (i.e., decreased binding of the last two neutrons), most probably indicates a neutron shell closure or mid-shell region. A subtle change in the slope of $S_{2 n}$ or a flattening in $S_{2 n}$ normally indicate collective effects. As a second derivative of nuclear masses, $G_{2 n}$ is useful to wipe out the smooth components. It is often used to show the strength of shell effect.

Similar to nuclear masses, nuclear charge radii are almost linearly dependent on neutron number between two neutron magic numbers, while a local minimum is found at magic numbers. Characteristic slope changes (kinks) of charge radii, especially the rapid increase of charge radii, have been found to be correlated to deformation effect. This is particularly striking in the $\mathrm{Hg}$ and Pt nuclei as will shown below.

$\delta V_{2 p-2 n}$ and $\overline{\delta R}_{p-n}$ involve at least four neighboring nuclei along two isotopic chains. They are thus useful to examine the local evolution of structure. More specifically, $\delta V_{2 p-2 n}$ values can help to explain and describe shell structure and orbit occupations near the Fermi surface, while deviations of $\overline{\delta R}_{p-n}$ from zero are found to be correlated to onset of deformation or shape coexistence. As we will show below, these different probes can be correlated and complimentary to each other.

Spectroscopic fingerprints - excited-state energies and $\gamma$-ray transition strengths — have played a leading role in the exploration of shape transitions, as they present patterns characteristic of the underlying nuclear shape. Here we choose the two most important observables: $E\left(2_{1}^{+}\right)$and $B(E 2)$. However, data on $E\left(2_{1}^{+}\right)$and $B(E 2)$ are much more sparse in comparison with the ground state properties as will be seen below.

The region of $Z \sim 40$ and $N \sim 60$ is known to be dominated by the sudden onset of deformation around $N=60$. Figure 1 shows the different observables discussed above. On addition of neutrons to the magic shell gap at $N=50$, nuclei undergo transitions from spherical to prolate (and oblate) deformations as $N=60$ is approached and crossed. The onset of deformation is clearly seen by a kink in $S_{2 n}$, and a rapid increase in nuclear size $R_{c h}$. This effect is more visible in $G_{2 n}$ and $S$ as a significant deviation from $G_{2 n} \approx 1$ and $S_{z} \approx 0$. The impact of the onset of deformation in $\delta V_{2 p-2 n}$ is similar to the shell closure at $N=50$, i.e., a significantly drop in magnitude from more than $0.6 \mathrm{MeV}$ to about $0.4 \mathrm{MeV}$. This is understandable because the empirical proton-neutron interaction is sensitive to the underlying nuclear levels, especially the valence nucleons, and both the shell closure and the rapid change in deformation can lead to similar changes in occupied single-particle orbits.

$\overline{\delta R}_{p-n}$ is particularly interesting. Very different from all the other observables, it is not sensitive to closed shells at all. This deviation might be due to the interplay of neutrons and protons, which somehow washes out the shell effects in charge radii. On the other hand, $\overline{\delta R}_{p-n}$ is apparently correlated with the onset of deformation, where there are large deviations from zero. Combined analysis of $\overline{\delta R}_{p-n}$ and the other observables like $S$, can even isolate the effects caused by closed shell closure and phase transitions.

The nuclides in the region of $Z \sim 64$ and $N \sim 90$ are centered on the stability line and thus are one of the most intensive and varied spectroscopic and theoretical 

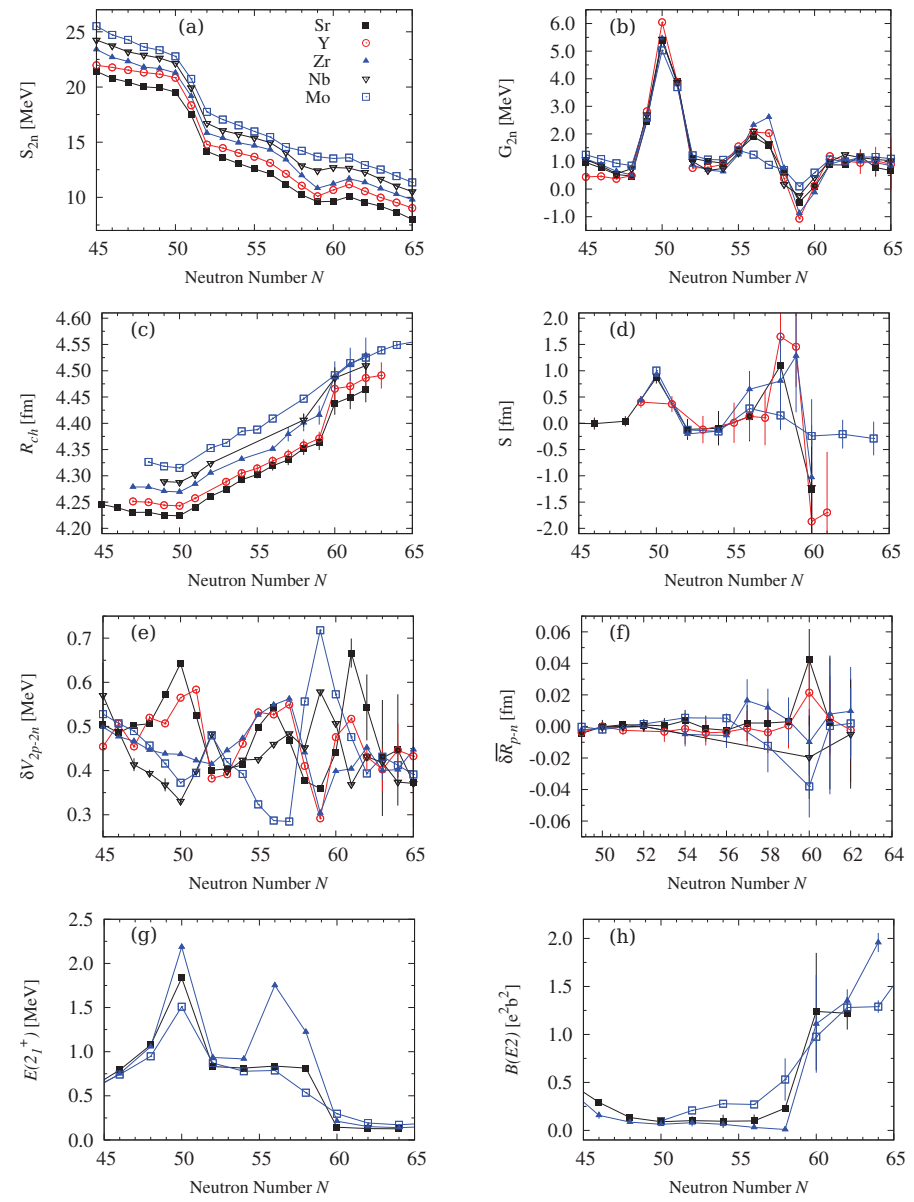

Figure 1. (a) Two-neutron separation energies $\left(S_{2 n}\right)$, (b) neutron shell gap $\left(G_{2 n}\right)$, (c) charge radii $\left(R_{c h}\right)$, (d) kink strength $(S)$, (e) effective interaction strength between last two valence neutrons and two valence protons $\left(\delta V_{2 p-2 n}\right)$, (f) a new charge radius relation $\left(\overline{\delta R}_{p-n}\right),(\mathrm{g})$ energy of the first-excited $2^{+}$state $\left(E\left(2_{1}^{+}\right)\right)$, and (h) transition probability between the first $2^{+}$and the $0^{+}$ground state $(B(E 2))$, for selected isotopic chains across the $N \sim 60$ region.

studies of collectivity. Figure 2 shows the systematics of all observables for this region. All observables exhibit a near identical pattern to that observed in the range of $(Z \sim 40, N \sim 60)$ except $\delta V_{2 p-2 n}$. The nuclear-mass region around $Z=82$ is characterized by the most extensive manifestation of shape coexistence. This effect can be immediately seen from extreme increases and "staggering" in charge radii and the relevant derivatives at the $N \sim 104$ region in Fig. 3. The sensitivity of $\overline{\delta R}_{p-n}$ to nuclear phase transitions is also shown. Correlations to shape coexistence are also shown in $S_{2 n}, G_{2 n}$ and $\delta V_{2 p-2 n}$, but it seems that $R_{c h}$ is much more sensitive to shape coexistence than $S_{2 n}$.

An interesting point from Figs. 1-3 is that the simple relation of $\overline{\delta R}_{p-n}=0$ holds to good precision except at occurrences of shape transitions. In other words, such relations offer a very useful feature in prediction of the root-mean-square (rms) charge radius of a given nuclide in terms of known charge radii of its neighboring nuclei. As an example, in Fig. 4 we show the difference between the predictions of charge radius using $\overline{\delta R}_{p-n}=0$ and experimental values. The predictions of $\overline{\delta R}_{p-n}=0$ are remarkably good throughout the nuclear chart, especially for nuclides heavier than Ni. However, larger deviations are seen at, e.g., $(Z \sim 40, N \sim 60),(Z \sim 55, N \sim 40)$, $(Z \sim 64, N \sim 90)$, and $Z \sim 80$, which are correlated with shape transitions. Moreover, the charge radii of $325 \mathrm{nu}-$ clides can be estimated as indicated in Fig. 4 within an accuracy of typically $0.01 \mathrm{fm}$. However, when sudden variances occur in nuclear shape, or where such changes are expected, one must be cautious about the predicitons of unknown charge radii. In these cases, it may be instructive to check the correlations with other ground and excited state observables.

\section{Summary}

To summarize, we examine possible hints of shape transition in ground state observables, i.e., masses and charge radii. In particular, we show that the recent proposed $\overline{\delta R}_{p-n}$ can be a good indicator for sudden changes in nuclear shape, and seems not to be affected by large shell closures. It is interesting to extend the analysis of $\overline{\delta R}_{p-n}$ in the near future over a wider range of isotopes, including exotic ones. Combined analysis of these different probes from mass and charge radii is always valuable, as these 

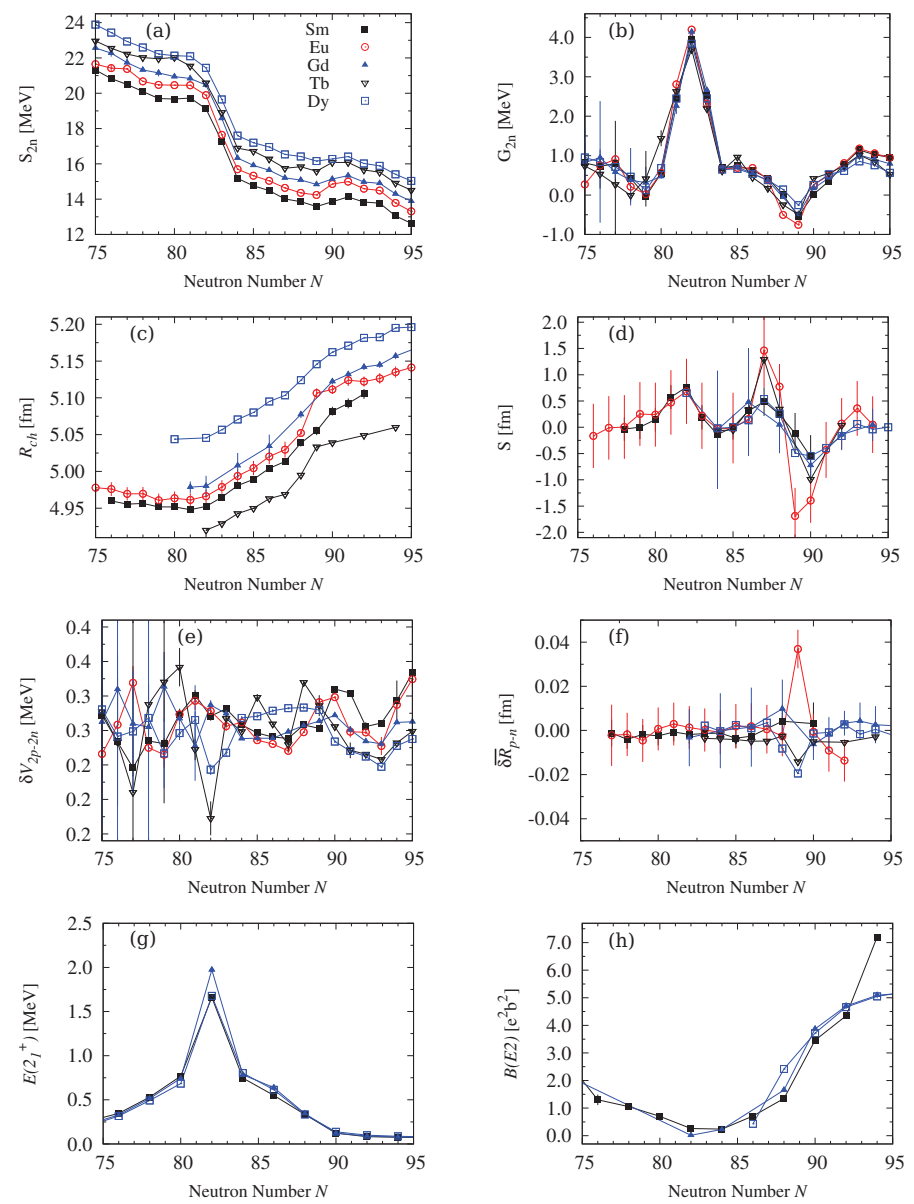

Figure 2. Same as Fig. 1, but for selected isotopic chains across the $N \sim 90$ region. To guide the eye, the large uncertainties of charge radii in $\mathrm{Tb}$ and $\mathrm{Dy}$ isotopic chains are not shown.

properties are often the first obtained for exotic nuclei in regions far from stability.

\section{Acknowledgements}

This work was supported partially by the National Natural Science Foundation of China (No. 11475014) and the Fundamental Research Funds for the Central Universities.

\section{References}

[1] P. Cejnar, J. Jolie, R. F. Casten, Rev. Mod. Phys. 82, 2155 (2010).

[2] K. Heyde and J. L. Wood, Rev. Mod. Phys. 83, 1467 (2011).

[3] I. Angeli et al., J. Phys. G 36, 085102 (2009).

[4] K. Blaum, Phys. Rep. 425, 1 (2006).

[5] B.H. Sun, Yu. A. Litvinov, I. Tanihata, Y. H. Zhang, Front. Phys. 10, 102102 (2015).

[6] R. B. Cakirli, R. F. Casten, K. Blaum, Phys. Rev. C 82, 061306(R) (2010).
[7] M. Wang, G. Audi, A. H. Wapstra, F. G. Kondev, M. MacCormick, X. Xu, and B. Pfeiffer, Chin. Phys. C 36, 1603 (2012).

[8] I. Angeli and K. P. Marinova, At. Data Nucl. Data Tables 99, 69 (2013).

[9] B. Pritychenko, M. Birch, B. Singh, M. Horoi, At. Data Nucl. Data Tables 107, 1 (2016).

[10] H. Jiang, G. J. Fu, Y. M. Zhao, and A. Arima, Phys. Rev. C 82, 054317 (2010).

[11] G. J. Fu, Y. Lei, H. Jiang et al., Phys. Rev. C 84, 034311 (2011).

[12] H. Jiang, G. J. Fu, B. Sun et al., Phys. Rev. C 85, 054303 (2012).

[13] G. J. Fu, J. J. Shen, Y. M. Zhao, and A. Arima, Phys. Rev. C 87, 044309 (2013).

[14] Y. Y. Cheng, Y. M. Zhao, and A. Arima, Phys. Rev. C 91, 024314 (2015).

[15] B. H. Sun, Y. Lu, J. P. Peng, C. Y. Liu, and Y. M. Zhao, Phys. Rev. C 90, 054318 (2014); ibid, Phys. Rev. C 91, 019902(E) (2015). 

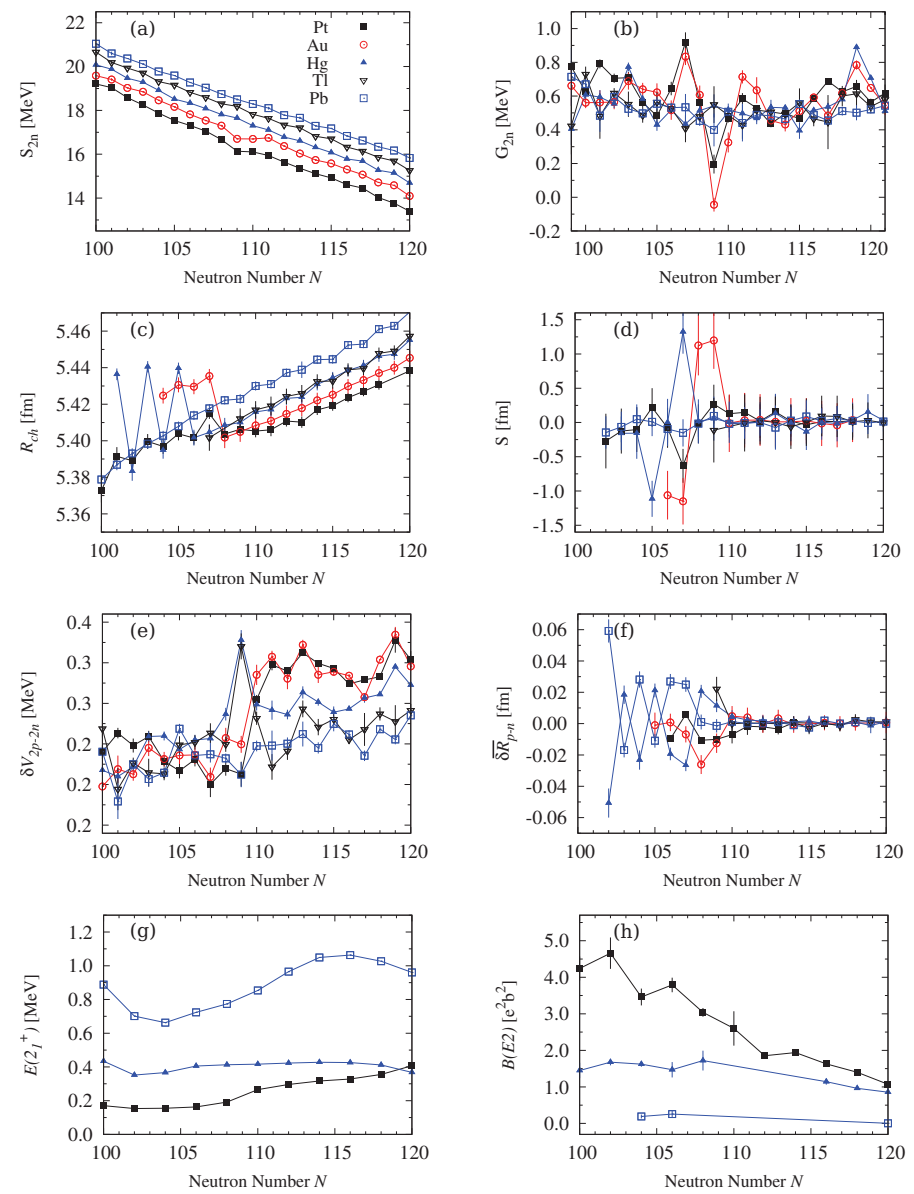

Figure 3. Same as Fig. 1, but for selected isotopic chains across the $Z \sim 82$ region. To guide the eye, the large uncertainties of $\overline{\delta R}_{p-n}$ charge radii in the ${ }^{184-187} \mathrm{Pt}$ isotopic chain are not shown.

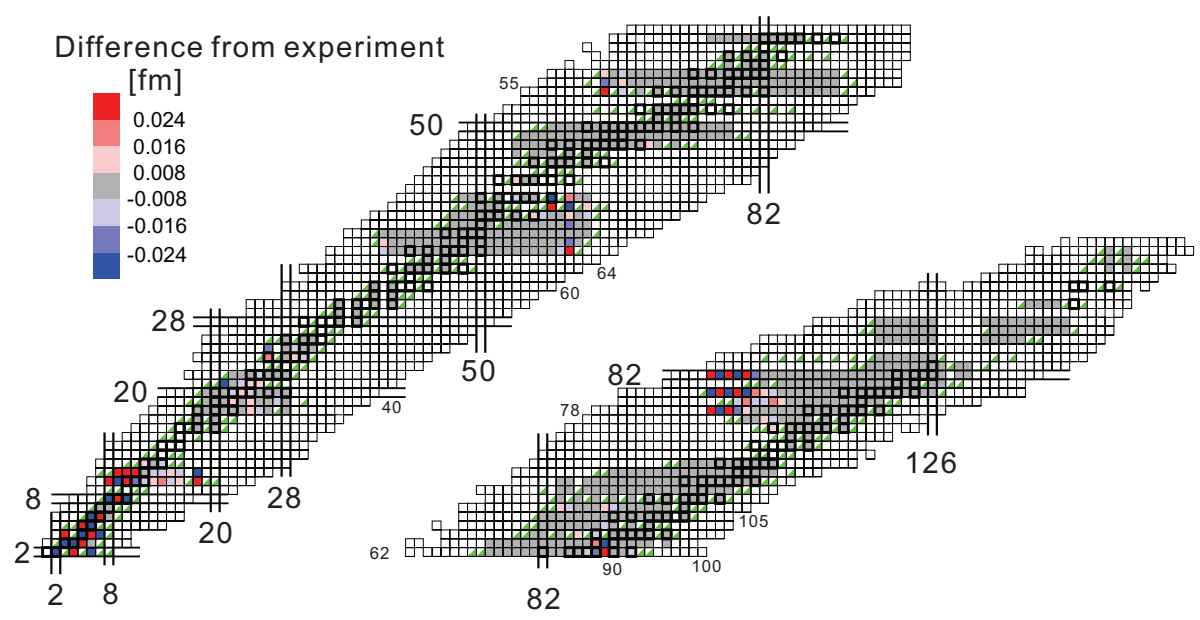

Figure 4. Chart of the nuclides showing the differences (colour coded, in fm) between the predictions of charge radii using $\overline{\delta R_{p-n}}=0$ and experimental values. The squares outlined in black indicate stable isotopes. The magic numbers are also indicated. 325 nuclides whose rms charge radius can be predicted within an accuracy around $0.01 \mathrm{fm}$ are marked by the green triangles. From Ref. [15]. 